\title{
Modeling the Relationship between Vibration Features and Condition Parameters Using Relevance Vector Machines for Health Monitoring of Rolling Element Bearings under Varying Operation Conditions
}

\author{
Lei Hu, ${ }^{1,2}$ Niao-qing Hu, ${ }^{1,2}$ Bin Fan, ${ }^{1,2}$ Feng-shou Gu, ${ }^{3}$ and Xiang-yi Zhang ${ }^{1,2}$ \\ ${ }^{1}$ Laboratory of Science and Technology on Integrated Logistics Support, National University of Defense Technology, \\ Changsha 410073, China \\ ${ }^{2}$ College of Mechatronics Engineering and Automation, National University of Defense Technology, Changsha 410073, China \\ ${ }^{3}$ School of Computing and Engineering, University of Huddersfield, Huddersfield HD1 3HD, UK
}

Correspondence should be addressed to Lei Hu; lake_hl@hotmail.com

Received 12 July 2014; Accepted 30 December 2014

Academic Editor: Yingwei Zhang

Copyright (C) 2015 Lei Hu et al. This is an open access article distributed under the Creative Commons Attribution License, which permits unrestricted use, distribution, and reproduction in any medium, provided the original work is properly cited.

\begin{abstract}
Rotational speed and load usually change when rotating machinery works. Both this kind of changing operational conditions and machine fault could make the mechanical vibration characteristics change. Therefore, effective health monitoring method for rotating machinery must be able to adjust during the change of operational conditions. This paper presents an adaptive threshold model for the health monitoring of bearings under changing operational conditions. Relevance vector machines (RVMs) are used for regression of the relationships between the adaptive parameters of the threshold model and the statistical characteristics of vibration features. The adaptive threshold model is constructed based on these relationships. The health status of bearings can be indicated via detecting whether vibration features exceed the adaptive threshold. This method is validated on bearings running at changing speeds. The monitoring results show that this method is effective as long as the rotational speed is higher than a relative small value.
\end{abstract}

\section{Introduction}

Rolling element bearing is a fundamental component of rotating machinery. Its fault is one of the most important causes for the failure of rotating machinery. Health monitoring of bearings aims at finding faults at their early stage which allows maintenance actions to be taken in time to prevent machinery from the failures. Conventional approaches are based on the assumption that the effect of bearing faults can be considered as load fluctuations in the time associated with the impact of rolling elements when passing through the defective zone [1]. These load fluctuations give periodic excitations of structural resonances as long as the rotational speed is constant. These periodic responses are named as the components of fault feature frequencies $[1,2]$. Traditional frequency based methods have been developed to extract the components of fault feature frequencies.

Rotating machine usually operates under varying load or speed conditions. For example, the output power and speed of an engine have to change with a large range to complete the slide, climbing, and landing process of an aircraft. In the cases where a machine operates under varying speeds or loads, its dynamics and vibrations exhibit nonstationary characteristics. Both the amplitudes and the frequencies of the vibration change with time $[1,3]$. Thus traditional frequency based methods are not applicable to such cases for health monitoring.

Great research efforts have been concentrated on nonstationary signal processing methods for health monitoring and fault diagnosis of rotating machinery. These methods 
include short time Fourier transform (STFT) [4], WignerVille distribution (WVD), wavelet analysis [5-7], and empirical mode decomposition (EMD) $[8,9]$. The STFT is to move a short time window along the vibration record and obtain the Fourier spectrum as a function of time shift. However, the frequency resolution is the reciprocal of the effective time window length; thus STFT could not give higher resolution both in time domain and frequency domain. The WVD obtains better local characteristics than STFT. But WVD is a nonlinear transform method and suffers from cross terms between the actual components. The wavelet analysis is to decompose the signal in terms of a family of "wavelets." The wavelet analysis possesses the multiscale character and gives a better time localization at high frequencies, and for that reason it has been widely applied to nonstationary signals analysis. But unfortunately, the wavelet analysis suffers from energy leakage in the vicinity of frequencies of interest due to the limited length of wavelet base function. The EMD decomposes the signal into a finite number of intrinsic mode functions (IMFs), which reflect the intrinsic of the signal. Therefore, the EMD method has been widely applied to nonstationary signal processing. However, the EMD also has problems, such as the end effect, the disturbances due to pseudo-information at low frequencies, and the IMF criterion. The order tracking method uses multiples of running speed, instead of absolute frequencies, as the frequency base. These multiples of running speed are named orders. Compared with other nonstationary signal processing methods, the order tracking method has advantages for analysis of speed-related vibration components, especially when rotational speed changes over a greater range $[3,10]$. However, angle domain signals are used for order tracking. And to obtain the angle domain signals, either additional hardware is needed to sample the vibration at constant increments of shaft angle, or additional software is needed to resample the vibration from time domain signals.

These signal processing methods provide ways to analyze vibration signal under varying operation conditions. Features that are extracted by these methods are assumed to vary with health status. But these nonstationary signal processing methods can be unavailable in some cases. For example, conditions could change sharply in short time, and the amplitudes of features extracted could be changed with both machine fault and the varying operation conditions. Thus how to recognize whether the changes of features are caused by faults or the varying operation conditions is difficult.

This paper aims at solving the health monitoring problem of bearings under varying operation conditions with pattern recognition method. We consider root mean square (RMS) $x^{\mathrm{R}}$ of the overall vibration, which can be computed easily, as a feature to indicate fault severity of bearings. RMS is a measurement of the effective energy content in a vibration signal. As vibration level generally increases when faults occur, RMS is well used for diagnostics and prognostics of rotating machines [11-13]. The proposed method is based on the relation functions between vibration features and condition parameters. The relationship functions are obtained with relevance vector machines (RVMs) and are used as adaptive parameters of threshold model. Since the adaptive parameters are continuous functions of condition parameters, the threshold model obtained is available at any operation conditions.

Relevance vector machines (RVMs) and support vector machines (SVMs) have been widely used in condition monitoring and fault diagnosis/prognosis [14-16]. But in previous works, RVMs/SVMs are mainly used for classification or regression. When RVMs/SVMs are used for classification, the inputs of RVMs/SVMs are vibration features and the outputs are pattern indexes. When RVMs/SVMs are used for regression, the inputs are vibration features and outputs are assessment of fault degree. The novelty of this paper is that RVMs are used to model the relationship between condition parameters and the statistics of vibration feature. The input of RVMs is condition parameter and the outputs are statistics of vibration feature. These statistics are used as adaptive parameters of fault monitoring model. And these adaptive parameters make the monitoring model adaptive and available under varying operation conditions.

\section{Methodology}

2.1. Adaptive Threshold Model. We select Gaussian threshold model (GTM) as the health monitoring model. The GTM is derived from Chebyshev's inequality [17]. For a feature $x$ that has Gaussian distribution, if its mean and standard deviation are $m$ and $d$, respectively, the following Chebyshev's inequality holds:

$$
P(|x-m| \geq k d) \leq k^{-2}, \quad \forall k>0 .
$$

The inequality means that the probability of $x \in(m-k d, m+$ $k d$ ) is bigger than $1-k^{-2} . k$ is called threshold tolerance. The above inequality is called three-sigma criterion when $k=3$. This model is also effective sometimes for features that are not Gaussian as long as the threshold tolerance is well optimized.

According to Chebyshev's inequality, for a given threshold tolerance $k$, the threshold interval of vibration RMS $x^{\mathrm{R}}$ can be constructed as $C=\left[m^{\mathrm{R}}-k d^{\mathrm{R}}, m^{\mathrm{R}}+k d^{\mathrm{R}}\right]$. As we are concerned about the upper bond only, a decision function can be obtained as $f\left(x^{\mathrm{R}}\right)=m^{\mathrm{R}}+k d^{\mathrm{R}}-x^{\mathrm{R}}$, which allows the monitoring to be implemented explicitly. $f\left(x^{\mathrm{R}}\right)$ is considered as the health index that indicates the health status of bearings; in particular, $f\left(x^{\mathrm{R}}\right) \geq 0$ means that the vibration level $x^{\mathrm{R}}$ is normal and the bearing is healthy, whereas $f\left(x^{\mathrm{R}}\right)<0$ means that $x^{\mathrm{R}}$ is abnormal and the bearing is faulty.

Here only rotational speed is addressed to simplify the problem. As the varying rotational speeds can also result in the change of vibration energy level, the adaptive GTM should be the function of both RMS $x^{\mathrm{R}}$ and the rotational speed $s$. Here we define the detecting function of the adaptive GTM as

$$
f\left(x^{\mathrm{R}}, s\right)=m^{\mathrm{R}}(s)+k d^{\mathrm{R}}(s)-x^{\mathrm{R}},
$$

where $m^{\mathrm{R}}(s)$ and $d^{\mathrm{R}}(s)$ are, respectively, mean and standard deviation of the RMS at rotational speed $s$ and $m^{\mathrm{R}}(s)+k d^{\mathrm{R}}(s)$ is the threshold of vibration level at rotational speed $s$. 
In order to apply the GTM to data at any rotational speeds, (2) should be a continuous function of $s$, which can be ensured as long as $m^{\mathrm{R}}(s)$ and $d^{\mathrm{R}}(s)$ are continuous functions. Two RVMs are used to fit $m^{\mathrm{R}}(s)$ and $d^{\mathrm{R}}(s)$. The first RVM, which is trained on samples $\left\{s(n), m^{\mathrm{R}}(n) \mid n=\right.$ $1,2, \ldots, N\}$, gives the rotational-speed-varying parameters $m^{\mathrm{R}}(s) . s(n)$ is the rotational speed of the $n$th sample. $m^{\mathrm{R}}(n)$ is the mean of vibration RMS at rotational speed $s(n) . N$ is the number of training samples. The second RVM, which is trained on samples $\left\{s(n), d^{\mathrm{R}}(n)\right\}$, gives the rotational-speedvarying parameters $d^{\mathrm{R}}(s) . d^{\mathrm{R}}(n)$ is the standard deviation of vibration RMS at rotational speed $s(n)$. These training samples are extracted from tests of normal bearings.

\subsection{Training Data Preparation}

2.2.1. Raw Data Collection. We adjust the rotational speed of healthy bearings in an interval $\left[s_{\min }, s_{\max }\right]$, specifically, we first raise the rotational speed from $s_{\min }$ to $s_{\max }$ and then lower it from $s_{\max }$ to $s_{\min }$. During the operation, vibration signals $v_{i}$ and rotational speed signals $r_{i}$ are sampled synchronously, and the subscript $i$ denotes that the signals are yielded during the $i$ th test, $i=1,2, \ldots, L$. Vibration signals are measured with accelerometers, and the rotational speed signals are measured with an optical encoder. $v_{i}$ and $r_{i}$ are the raw data required in the proposed method.

2.2.2. Estimation of Rotational Speed and Vibration Level. The raw rotational speed signals $r_{i}$ collected with the optical encoder are series of pulses. One pulse is generated for each revolution. We search the raw data for points that satisfy $r_{i}(j) \leq 0$ and $r_{i}(j+1) \geq 0$. Then the zero-crossing time $t_{j}$ can be estimated with linear interpolation by

$$
\frac{0-r_{i}(j)}{t_{j}-t(j)}=\frac{r_{i}(j+1)-r_{i}(j)}{t(j+1)-t(j)},
$$

where $t(j)$ and $t(j+1)$ are the time instants for $r_{i}(j)$ and $r_{i}(j+$ $1)$, respectively. Then the speed $s_{j}$ at time $t_{j}$ can be estimated by $s_{j}=60 /\left(t_{j}-t_{j-1}\right)$, in which $t_{j}$ is the $j$ th zero-crossing time and $t_{j-1}$ is the $(j-1)$ th zero-crossing time.

The vibration RMS $x_{j}^{\mathrm{R}}$ at time $t_{j}$ is estimated with the vibration points that are sampled in the time interval $\left(t_{j-1}, t_{j}\right]$.

\subsubsection{Statistical Characteristics Estimation of RMS. We sup-} pose $\left\{s_{i j}\right\}$ and $\left\{x_{i j}^{\mathrm{R}}\right\}$ to be the rotational speed series and the RMS series that are obtained from the $i$ th test. It should be noted that the series $\left\{s_{i j}\right\}$ of different tests are different. In order to compute the statistical parameters of RMS at different rotational speeds, we need to resample $\left\{x_{i j}^{\mathrm{R}}\right\}$ to make them share a common rotational speed series $\{s(n) \mid n=$ $1,2, \ldots, N\}$. The series $s(n)$ has equal speed increments.

The corresponding RMS series $\left\{x_{i}^{\mathrm{R}}(n)\right\}$ of the $i$ th test can be yielded with linear interpolation. We search the series $\left\{s_{i j}\right\}$ for the points that satisfy $\left(s_{i j}-s(n)\right)\left(s_{i(j+1)}-s(n)\right) \leq 0$. The corresponding RMS can be estimated by

$$
\frac{x_{i}^{\mathrm{R}}(n)-x_{i j}^{\mathrm{R}}}{x_{i(j+1)}^{\mathrm{R}}-x_{i}^{\mathrm{R}}(n)}=\frac{s(n)-s_{i j}}{s_{i(j+1)}-s(n)} .
$$

The RMS series $\left\{x_{i}^{\mathrm{R}}(n)\right\}$ yielded shares a common rotational speed coordinate.

We carry out $L$ times of tests and yield $L$ series $\left\{x_{i}^{\mathrm{R}}(n) \mid\right.$ $i=1,2, \ldots, L\}$. The mean series and the standard deviation series of RMS can be yielded by

$$
\begin{aligned}
m^{\mathrm{R}}(n) & =\frac{1}{L} \sum_{i=1}^{L} x_{i}^{\mathrm{R}}(n), \\
d^{\mathrm{R}}(n) & =\sqrt{\frac{1}{L-1} \sum_{i=1}^{L}\left(x_{i}^{\mathrm{R}}(n)-m^{\mathrm{R}}(n)\right) .}
\end{aligned}
$$

2.3. Adaptive Parameter Estimation. If we replace $\left(m^{\mathrm{R}}(s)\right.$, $\left.d^{\mathrm{R}}(s)\right)$ in (2) with $\left\{m^{\mathrm{R}}(n), d^{\mathrm{R}}(n) \mid n=1,2, \ldots, N\right\}, N$ detectors will be generated and these detectors are only applicable to $x^{\mathrm{R}}$ whose corresponding rotational speed is $s(n)$. Such detectors are not practical. In order to solve this problem, we introduce two RVMs to generate the detector of (2). The first RVM is trained on the set $\left\{s(n), m^{\mathrm{R}}(n)\right\}$ and gives continuous function $m^{\mathrm{R}}(s)$. The second one is trained on the set $\left\{s(n), d^{\mathrm{R}}(n)\right\}$ and gives continuous function $d^{\mathrm{R}}(s)$. Putting $m^{\mathrm{R}}(s)$ and $d^{\mathrm{R}}(s)$ into (2) yields the adaptive GTM, which is applicable at any rotational speed. A brief introduction about RVM regression will be given in Section 2.4.

2.4. Relevance Vector Machines for Regression. RVMs models were originally proposed by Tipping $[18,19]$. RVMs share the functional form with the popular support vector machines (SVMs). RVMs exploit a probabilistic Bayesian learning framework and utilize dramatically fewer kernel functions than comparable SVMs. RVMs have a number of additional advantages. These advantages include the benefits of probabilistic predictions, automatic estimation of parameters, and the facility to utilize arbitrary kernel functions. In this section, a brief outline of the RVMs theory is present for applying it to the bearing datasets. The details of RVM can be found in $[18,19]$.

Given a dataset of training pairs $\left\{\mathbf{x}_{i}, y_{i}\right\}_{i=1}^{N}$, where $\mathbf{x}_{i}$ is the input feature vector, $y_{i}$ is the target output of $\mathbf{x}_{i}$, and $N$ is the number of samples. RVMs make predictions based on the model of (6) that shares a functional form with SVMs. Consider

$$
\tilde{y}_{i}=\sum_{n=1}^{N} w_{n} K\left(\mathbf{x}_{i}, \mathbf{x}_{n}\right)+w_{0}=\mathbf{w}^{\mathrm{T}} \mathbf{K}+w_{0},
$$

where $\mathbf{w}=\left[w_{1}, \ldots, w_{N}\right]^{\mathrm{T}}$ is the weight vector, $\mathbf{K}=\left[K\left(\mathbf{x}_{i}, \mathbf{x}_{1}\right)\right.$, $\left.\ldots, K\left(\mathbf{x}_{i}, \mathbf{x}_{N}\right)\right]^{\mathrm{T}}$ is the kernel function vector, and $w_{0}$ is 
the offset. Gaussian kernel function is generally selected. Consider

$$
K\left(\mathbf{x}_{i}, \mathbf{x}_{j}\right)=\exp \left(-\frac{\left\|\mathbf{x}_{i}-\mathbf{x}_{j}\right\|^{2}}{w^{2}}\right),
$$

where $w$ is the basis width of the kernel.

For a test sample $\mathbf{x}_{i}$ whose output target $y_{i}$ is unknown, the trained RVMs give $\tilde{y}_{i}$ as the predicted value of $y_{i} \cdot y_{i}$ is class label in cases where RVMs are used for classification, and $y_{i}$ is real value in cases where RVMs are used for regression.

The output $\tilde{y}_{i}$ can be regarded as output target polluted with Gaussian noise. Due to the assumption of independence of the $y_{i}$, by adopting the Bernoulli distribution for $P(y \mid \mathbf{x})$, the likelihood of the dataset $\left\{\mathbf{x}_{i}, y_{i}\right\}_{i=1}^{N}$ can then be written as

$$
p\left(\mathbf{y} \mid \mathbf{w}, \sigma^{2}\right)=\left(2 \pi \sigma^{2}\right)^{-N / 2} \exp \left\{-\frac{1}{2 \sigma^{2}}\|\mathbf{y}-\mathbf{\Phi} \mathbf{w}\|^{2}\right\},
$$

where $\mathbf{y}=\left[y_{1}, \ldots, y_{N}\right], \mathbf{w}=\left[w_{0}, \ldots, w_{N}\right]$, and $\boldsymbol{\Phi}$ is the $N \times$ $(N+1)$ matrix with $\boldsymbol{\Phi}_{i j}=K\left(\mathbf{x}_{i}, \mathbf{x}_{j-1}\right)$ and $\boldsymbol{\Phi}_{i 1}=1$.

In order to overcome overfitting, RVMs adopt a Bayesian perspective, and zero-mean Gaussian prior distribution is used to introduce an individual hyperparameter $\alpha_{i}$ on each weight $w_{i}$. The prior distribution of weights then can be written as

$$
p(\mathbf{w} \mid \boldsymbol{\alpha})=\prod_{n=1}^{N} N\left(w_{n} \mid 0, \alpha_{i}^{-1}\right)=\left(\frac{\boldsymbol{\alpha}}{2 \pi}\right)^{(N+1) / 2} \exp \left(-\frac{\boldsymbol{\alpha}}{2} \mathbf{w}^{2}\right),
$$

where $\boldsymbol{\alpha}=\left[\alpha_{0}, \ldots, \alpha_{N}\right]$. The posterior distribution over the weights is then obtained from Bayes' rule:

$$
\begin{aligned}
p & \left(\mathbf{w} \mid \mathbf{y}, \boldsymbol{\alpha}, \sigma^{2}\right) \\
& =(2 \pi)^{-(N+1) / 2}|\boldsymbol{\Sigma}|^{-1 / 2} \exp \left\{-\frac{1}{2}(\mathbf{w}-\boldsymbol{\mu})^{\mathrm{T}} \boldsymbol{\Sigma}^{-1 / 2}((\mathbf{w}-\boldsymbol{\mu}))\right\}
\end{aligned}
$$

with $\boldsymbol{\Sigma}=\left(\boldsymbol{\Phi}^{\mathrm{T}} \mathbf{B} \boldsymbol{\Phi}+\mathbf{A}\right)^{-1}, \boldsymbol{\mu}=\boldsymbol{\Sigma} \boldsymbol{\Phi}^{\mathrm{T}} \mathbf{B y}$, where $\mathbf{A}=\operatorname{diag}\left(\alpha_{0}\right.$, $\left.\alpha_{1}, \ldots, \alpha_{N}\right)$ and $\mathbf{B}=\sigma^{-2} \mathbf{I}_{N}$.

By integrating out the weights, the marginal likelihood can be obtained for the hyperparameters:

$$
\begin{aligned}
p\left(\mathbf{y} \mid \boldsymbol{\alpha}, \sigma^{2}\right)= & (2 \pi)^{-N / 2}\left|\mathbf{B}^{-1}+\boldsymbol{\Phi} \mathbf{A}^{-1} \boldsymbol{\Phi}^{\mathrm{T}}\right|^{-1 / 2} \\
& \cdot \exp \left\{-\frac{1}{2} \mathbf{y}^{\mathrm{T}}\left(\mathbf{B}^{-1}+\boldsymbol{\Phi} \mathbf{A}^{-1} \boldsymbol{\Phi}^{\mathrm{T}}\right)^{-1} \mathbf{y}\right\} .
\end{aligned}
$$

Tipping released a Matlab software package named SparseBayes Version 2.0. The software computes the posterior distribution over the hyperparameters $\boldsymbol{\alpha}$ and returns their most-probable values by maximizing the marginal likelihood function equation (11). As many of the weights are mathematically set to zero, a lower number of kernel functions are used to construct the predictive model. This results in a sparse model, which can generalize well and is fast to compute. The pairs of data $\left(\mathbf{x}_{i}, y_{i}\right)$ with nonzero weights are called relevance vectors.

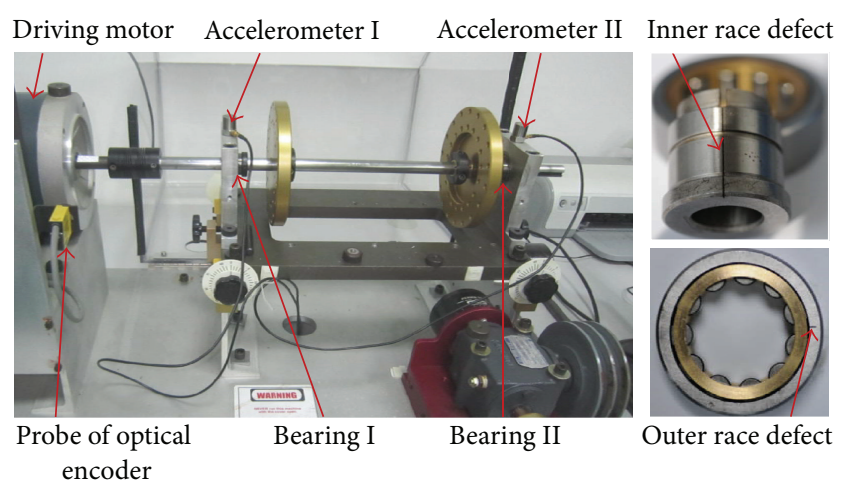

FIGURE 1: Test rig of machinery fault simulation and bearings with defects.

2.5. Discussions. The proposed health monitoring method concerns the selection of two parameters, in particular the threshold tolerance $k$ in (2) and the basis width $w$ in Gaussian kernel equation (7). The threshold tolerance $k$ is determinative to balance false negative and false positive. According to (2), the larger the $k$ is, the wider the threshold interval is and hence the smaller the probability that the normal samples exceed the threshold intervals, which means that the system has fewer false negatives. Conversely, the smaller the $k$ is, the narrower the threshold interval is and the smaller the probability that the novel samples are located in the threshold intervals, which means that the system is more sensitive. So $k$ can be set with cross-validation as follows: (1) initialize $k$ to be 3 or any value; (2) if monitoring results of historical data show unacceptable false negative alarms, increase $k$; (3) on the contrary, if monitoring results of historical data show unacceptable false positive rate, reduce $k$; (4) false negative rate and false positive rate cannot be reduced simultaneously, and a balance between them should be reached [17].

The basis width $w$ is a determinative parameter for RVMs. The larger the basis width is, the sparser the RVMs are and fewer points are relevance vectors. On the contrary, the smaller the basis width is, the more the relevance vectors will be selected, and the RVMs have worse generalization. Choosing of basis width is experiential. Cross-validation results can be referred to for choosing of basis width.

\section{Experiment Verification}

3.1. Monitoring Model Construction. The proposed method was applied to a test rig of machinery fault simulation, as shown in Figure 1. In order to compute the mean series $\left\{m^{\mathrm{R}}(n)\right\}$ and the standard deviation series $\left\{d^{\mathrm{R}}(n)\right\}$ as training samples, 20 tests of healthy bearings were carried out on the test rig. The rotational speed interval of the motor is set to be [200 1600] rpm. The vibration signals and rotational speed signals were collected synchronously. And vibration RMS and rotational speeds were computed for each revolution. Figure 2 shows the rotational speeds and the vibration RMS values of a healthy bearing obtained by a test. It can be seen from Figure 2 that although the bearing is healthy, vibration energy level changes greatly at different rotational speeds. At the 


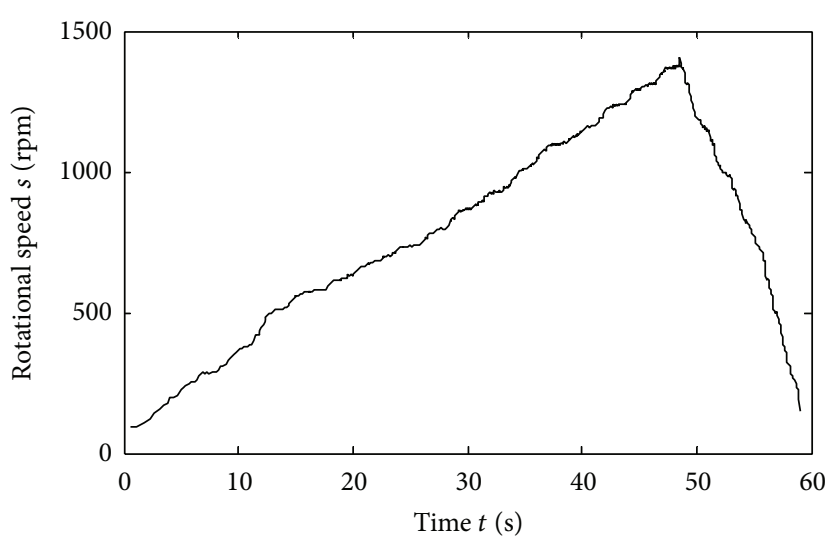

(a)

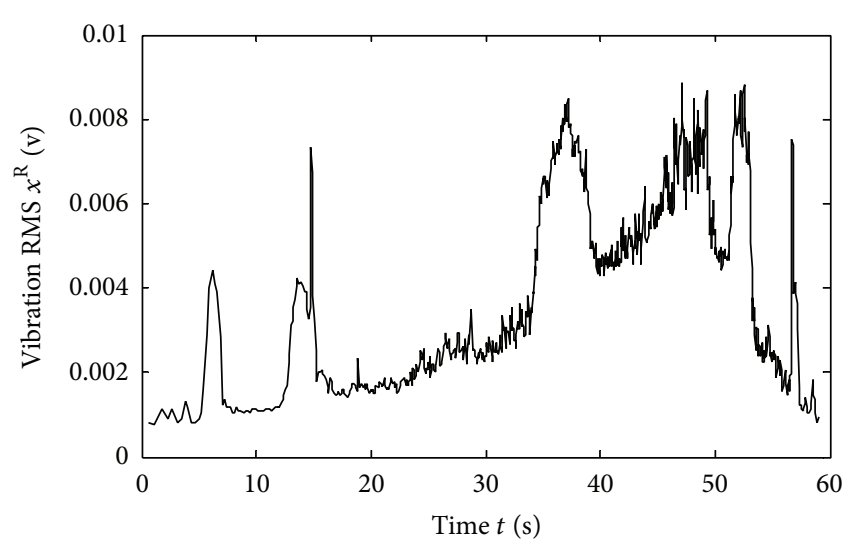

(b)

FIGURE 2: Varying of rotational speeds and vibration level of a healthy bearing: (a) rotational speeds, (b) vibration RMS.

same time, the fault status may only lead to a little change in system vibration response. Thus it is difficult to identify whether the change of vibration energy is caused by faults or varying operation conditions.

As mentioned in Section 2.2.3, different tests generate different rotational speed series. In order to compute the statistical mean and standard deviation of RMS at different rotational speeds, a new rotational speed series with equal speed increments is created first, and then the RMS series corresponding to the new speed series is estimated for each test. As the rotational speeds range from $200 \mathrm{rpm}$ to $1600 \mathrm{rpm}$, the new speed series will be $\{s(n), n=1,2, \ldots, N\}=$ $\{203,209,215, \ldots, 1397\}$ when we set $N=200$. The RMS series of each test corresponding to the rotational speed series can be estimated with the linear interpolation method introduced in Section 2.2.3.

Selection of the threshold tolerance $k$ using cross-validation requires fault samples for computation of false positive rates. In order to generate fault samples, tests of healthy bearings and bearings with seeded inner race defects and outer race defects were carried out, as shown in Figure 1. The bearings with seeded defects were installed in the position of bearing I. The vibration RMS values and rotational speeds of testing samples were also computed with each circle the machine rotates.

20 RMS series $\left\{x_{i}^{\mathrm{R}}(n), i=1,2, \ldots, 20, n=1,2, \ldots, 200\right\}$ were generated from 20 tests of healthy bearings. Then the mean series $\left\{m^{\mathrm{R}}(n)\right\}$ and standard deviation series $\left\{d^{\mathrm{R}}(n)\right\}$ were estimated with (5). $\left\{s(n), m^{\mathrm{R}}(n)\right\}$ and $\left\{s(n), d^{\mathrm{R}}(n)\right\}$ are training samples of RVMs. The parameter $m^{\mathrm{R}}(s)$ of the adaptive GTM was yielded by training a RVM with $\left\{s(n), m^{\mathrm{R}}(n)\right\}$, and $d^{\mathrm{R}}(s)$ was yielded by training another RVM with $\left\{s(n), d^{\mathrm{R}}(n)\right\}$. In order to validate the regression results and to select the basis width $w$ with cross-validation, we need to generate testing samples of RVMs. We generated a new rotational speed series $\{s(m)\}=\{206,212,218, \ldots, 1394\}$, and the corresponding mean series $\left\{m^{\mathrm{R}}(m)\right\}$ and standard deviation series $\left\{d^{\mathrm{R}}(m)\right\}$ were yielded in the same way as above. $\left\{s(m), m^{\mathrm{R}}(m)\right\}$ and $\left\{s(m), d^{\mathrm{R}}(m)\right\}$ are testing samples of RVMs.
Selection of the basis width $w$ is vital for RVM regression. For cross-validation of $w$, we define training error $E_{1}$ and testing error $E_{2}$ of RVM, respectively, as

$$
\begin{aligned}
& E_{1}=\frac{1}{N} \sum_{i=1}^{N} \frac{\left|y_{i}^{r}-\tilde{y}_{i}^{r}\right|}{y_{i}^{r}}, \\
& E_{2}=\frac{1}{N} \sum_{i=1}^{N} \frac{\left|y_{i}^{e}-\widetilde{y}_{i}^{e}\right|}{y_{i}^{e}},
\end{aligned}
$$

where $y_{i}^{r}$ is the real target of a training sample, $\tilde{y}_{i}^{r}$ is the estimated output given by RVM, $y_{i}^{e}$ is the real target of a testing sample, and $\widetilde{y}_{i}^{e}$ is the estimated output given by RVM.

The cross-validation results of the first RVM, which is used for regression of $m^{\mathrm{R}}(s)$, are shown in Figure 3 . Figure 3(a) illustrates the training error and testing error with varying basis width in the interval $[2,70]$. It can be seen that both the training error and the testing error decrease dramatically with the increase of $w$ at the beginning. If $w$ becomes bigger than 6 , the training error and the testing error turn out to increase slowly with the increase of $w$. Figure 3(b) illustrates the number of relevance vectors. It can be seen that the number of relevance vectors decreases with the increase of $w$. We can infer from Figure 3 that when $w$ takes values in the interval $[30,70]$, both the testing error and the training error are smaller than 0.09 , and the model is relatively sparse as the number of relevance vectors is smaller than 38 . We set the basis width to be $w=48$; in such case, both the training error and the testing error are 0.06 , and the number of relevance vectors is 24 . The cross-validation results of the second RVM, which is used for regression of $d^{\mathrm{R}}(s)$, are shown in Figure 4. Similarly, we can infer from Figure 4 that $w$ can take values in the interval $[20,70]$. Once $w=28$ is chosen, both the training error and the testing error are 0.06 , and the number of relevance vectors is 34 .

The regression and test results of the parameters $m^{\mathrm{R}}(s)$ and $d^{\mathrm{R}}(s)$ are, respectively, shown in Figures 5 and 6. It can be seen from Figure 5 that vibration RMS values have a general uptrend when rotational speeds increase. Vibration level reaches the local peak when the rotational speeds are 


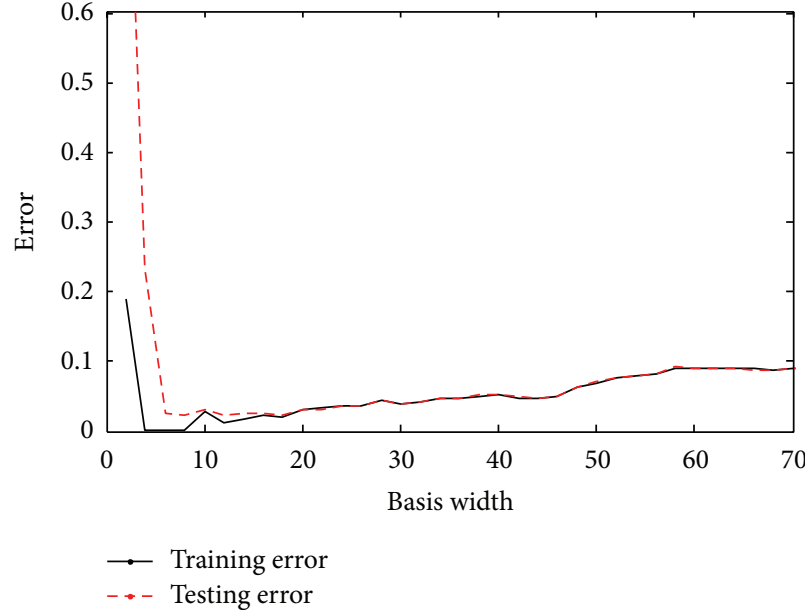

(a)

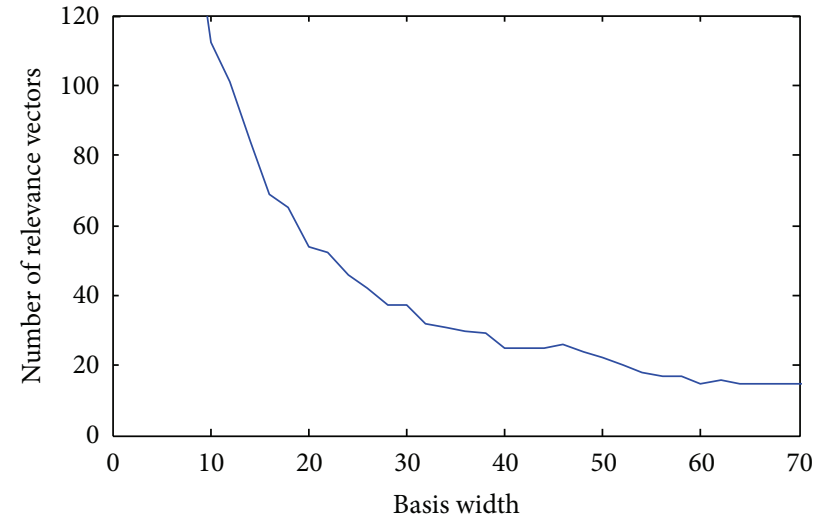

(b)

FIGURE 3: Regression characteristics of $m^{\mathrm{R}}(s)$ with different basis width: (a) regression error; (b) number of relevance vectors.

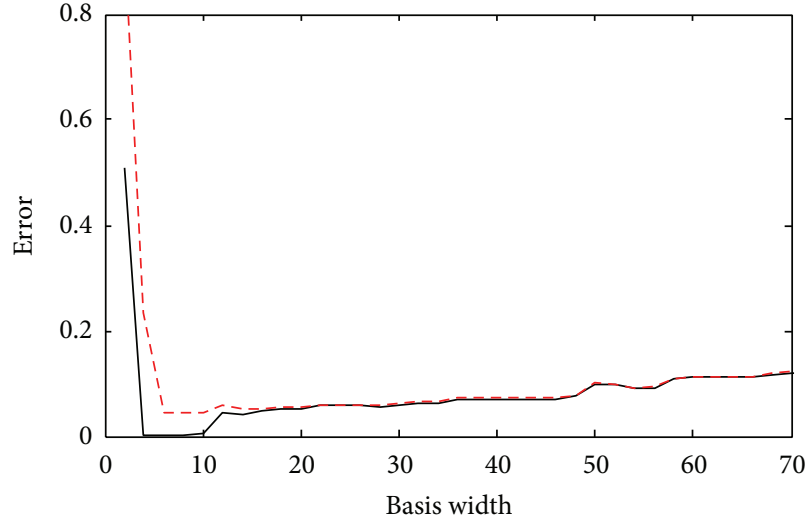

$\rightarrow$ Training error

- - Testing error

(a)

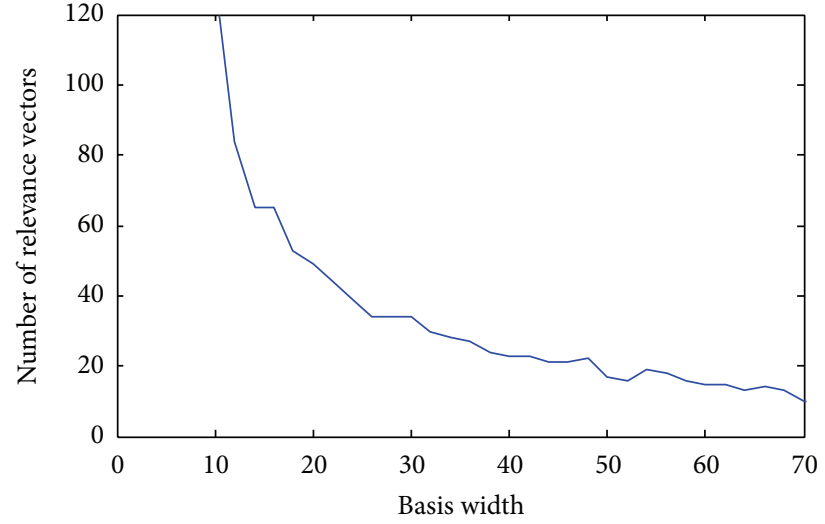

(b)

Figure 4: Regression characteristics of $d^{\mathrm{R}}(s)$ with different basis width: (a) regression error; (b) number of relevance vectors.

around 4 particular values, 257, 527, 1037, and $1349 \mathrm{rpm}$. The regression precision of $m^{\mathrm{R}}(s)$ is high as few testing samples or training samples are far away from the regression curve. It can be seen from Figure 6 that the standard deviation of vibration RMS is also higher when the rotational speeds are around the 4 particular values. The adaptive parameter $d^{\mathrm{R}}(s)$ is also well fitted. There are only several testing samples and training samples that are far away from the regression curve, and these samples are around the 4 particular rotational speeds.

We let the threshold tolerance $k$ change in the interval $[0.5,10]$ and apply the corresponding detectors to testing samples. The false negative rates and the false positive rates yielded by detectors with different threshold tolerance are shown in Figure 7. It can be seen that false negative rate decreases with the increase of $k$. The false negative rate is close to 0.04 when $k$ increases to 3.5. The false positive rate increases with the increase of $k$. The false positive rate is 0.21 when $k=3.5$. As high false negative rate is generally more intolerable than high false positive rate for field engineers, a relative bigger $k=3.5$ is an optimized selection.

3.2. Monitoring Results. Figure 8 shows the monitoring results of a healthy bearing during Test 18 . The rotational speeds of the tested bearing decreased from $1400 \mathrm{rpm}$ to $200 \mathrm{rpm}$ in 20.0 seconds. The total number of the extracted samples is 215 . Figure 8(a) describes the vibration RMS values and their thresholds at different time and rotational speeds. Figure 8(b) shows the health indexes at different rotational speeds. There are 8 samples whose RMS values exceed their thresholds. Two of these 8 samples are at around $471 \mathrm{rpm}$, another 5 are at the interval [941 993] rpm, and the other one is at round $1202 \mathrm{rpm}$. These 8 samples, whose health indexes are negative as shown in Figure 8(b), are false negatives. The false negative rate is $8 / 215 \approx 0.037$. 


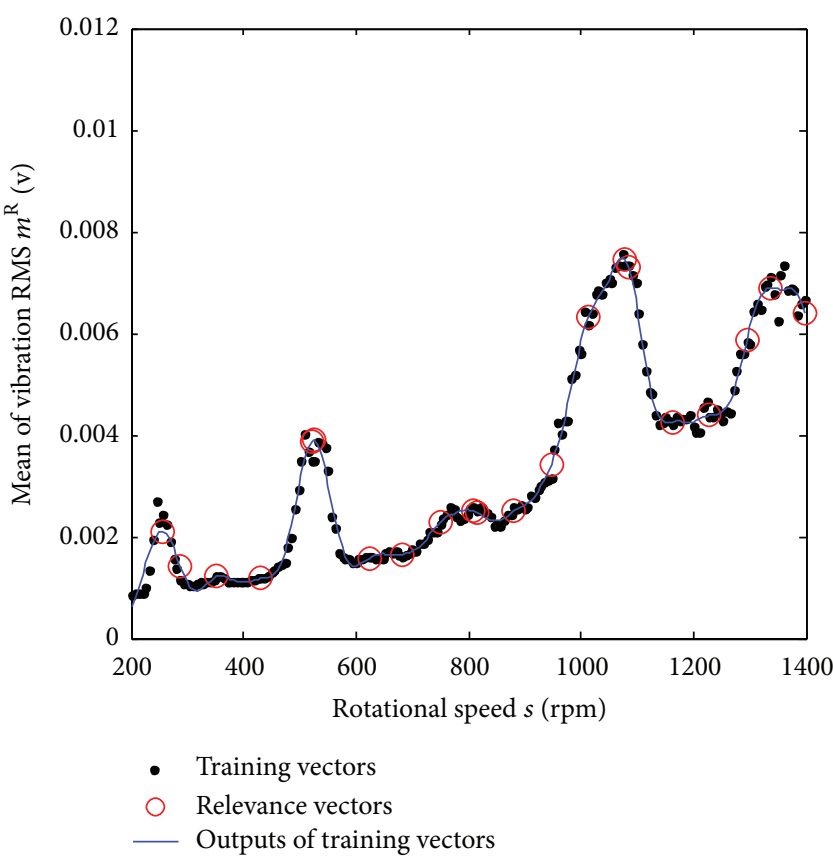

(a)

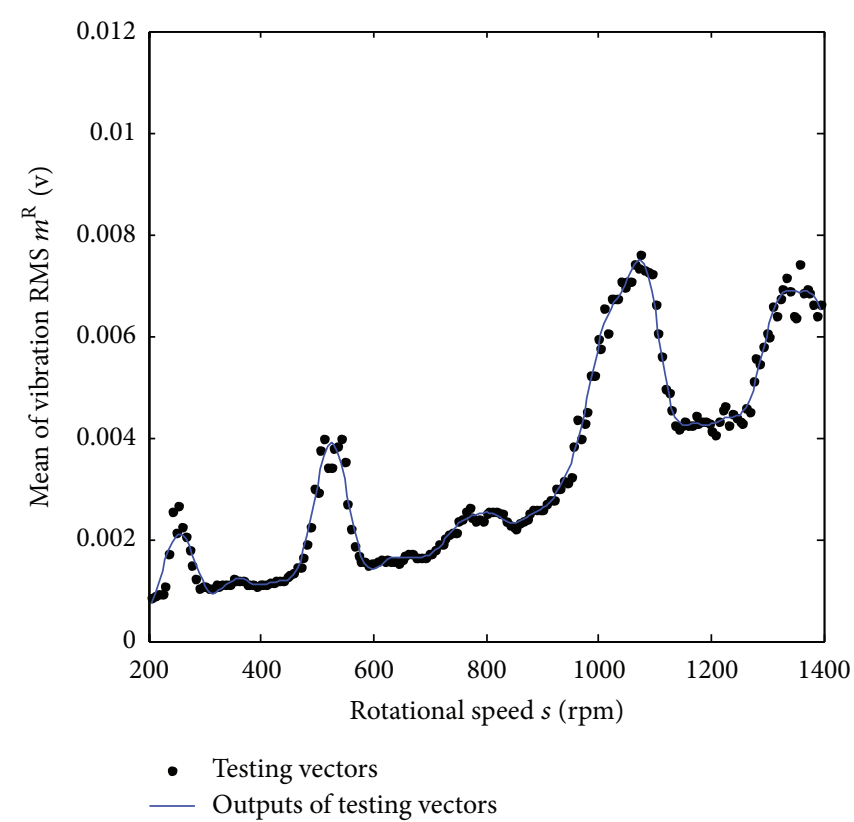

(b)

FIGURE 5: Regression and test results of parameter $m^{\mathrm{R}}(s)$ : (a) regression results; (b) testing results.

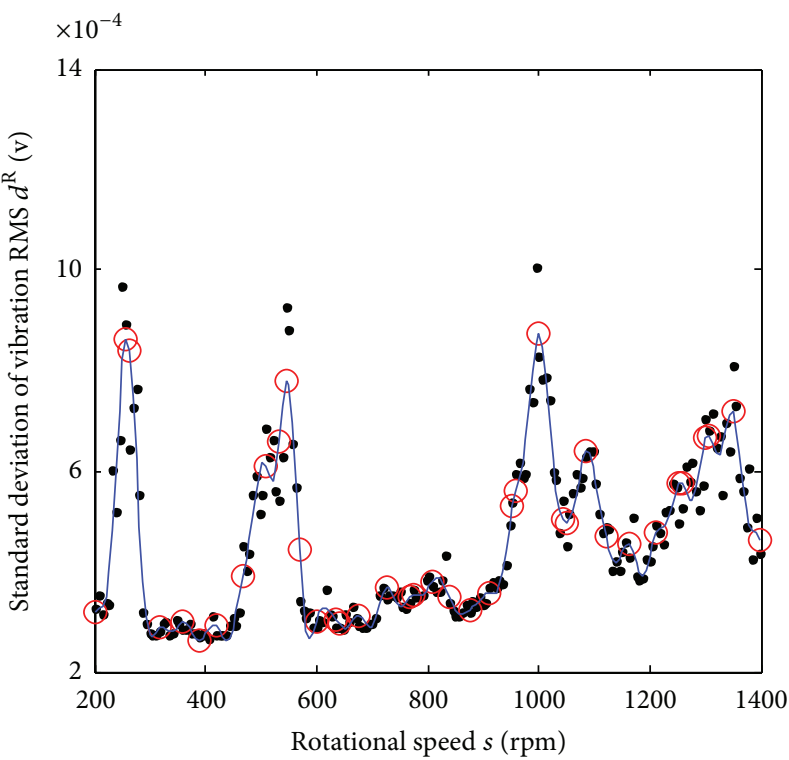

- Training vectors

- Relevance vectors

(a)

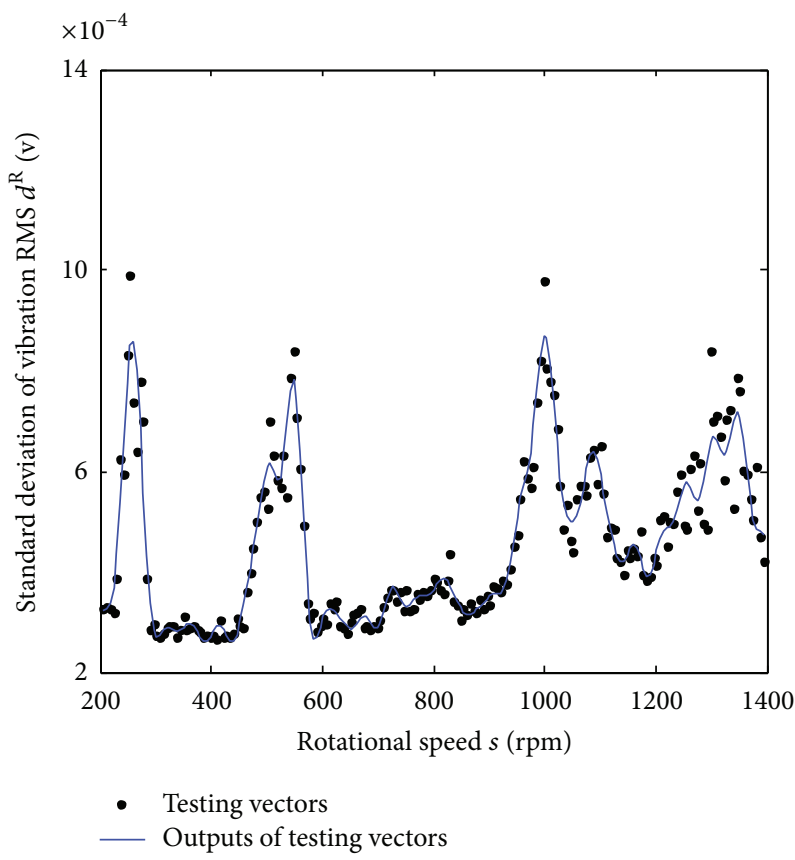

(b)

FIGURE 6: Regression and test results of parameter $d^{\mathrm{R}}(s)$ : (a) regression results; (b) testing results.

Figure 9 shows the monitoring results of a healthy bearing during Test 19. Test 19 lasted 59.1 seconds, during which the rotational speeds increased from $200 \mathrm{rpm}$ to $1400 \mathrm{rpm}$. The number of the extracted samples is 669. There is only a sample whose RMS value exceeds its corresponding thresholds as shown in Figure 9(a). The sample is at around $282 \mathrm{rpm}$. The health index of the sample is negative as shown in Figure 9(b). This sample is false negative. The false negative rate is $1 / 669 \approx$ 0.002 .

Figure 10 shows the monitoring results of a faulty bearing during Test 14 . The rotational speeds decreased from $1271 \mathrm{rpm}$ to $200 \mathrm{rpm}$ in 55.7 seconds. It can be seen that 


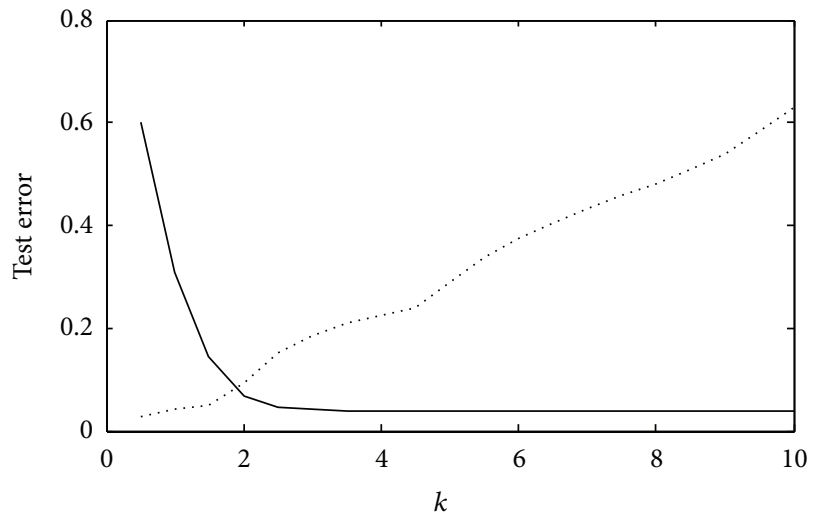

False positive rate

False negative rate

FIGURE 7: Change of test error at different threshold tolerance.

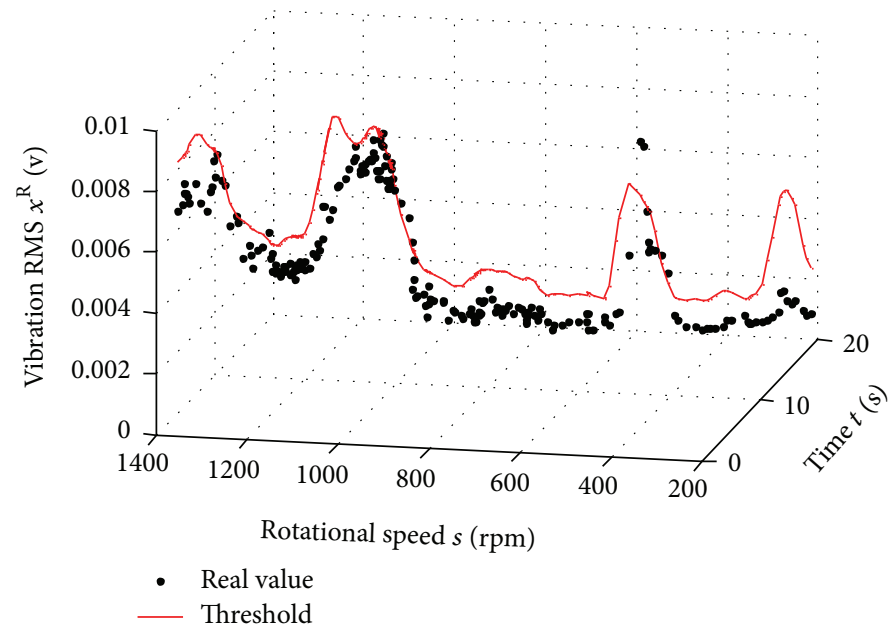

(a)

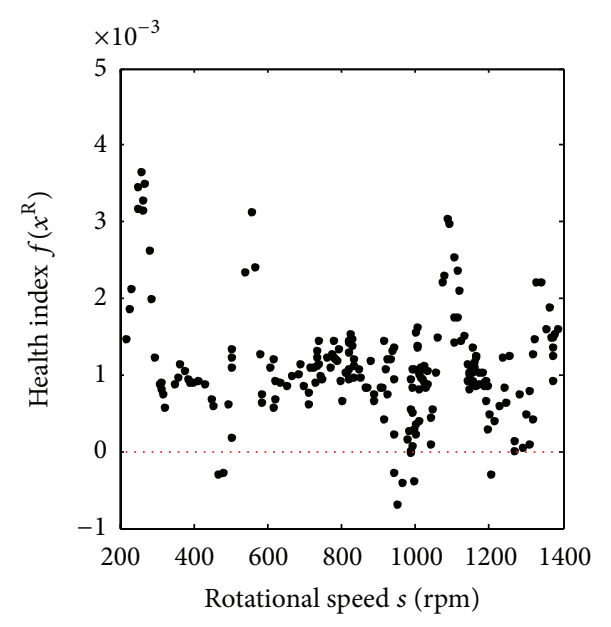

(b)

FIgURE 8: The monitoring results of a healthy bearing during Test 18.

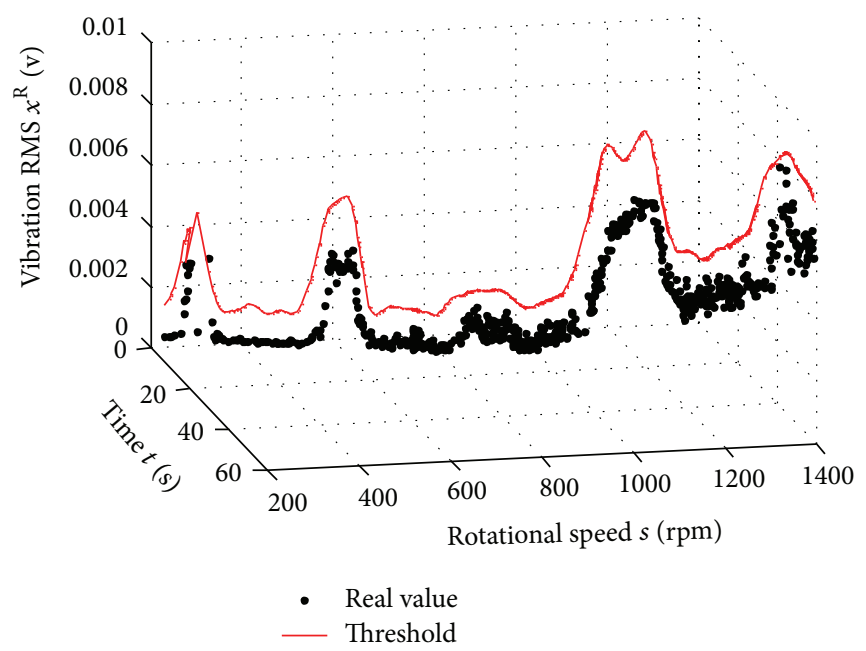

(a)

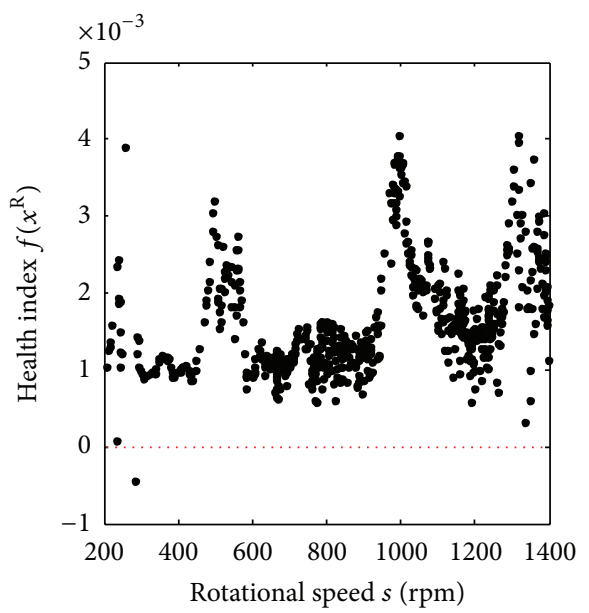

(b)

FIgURE 9: The monitoring results of a healthy bearing vibration during Test 19. 


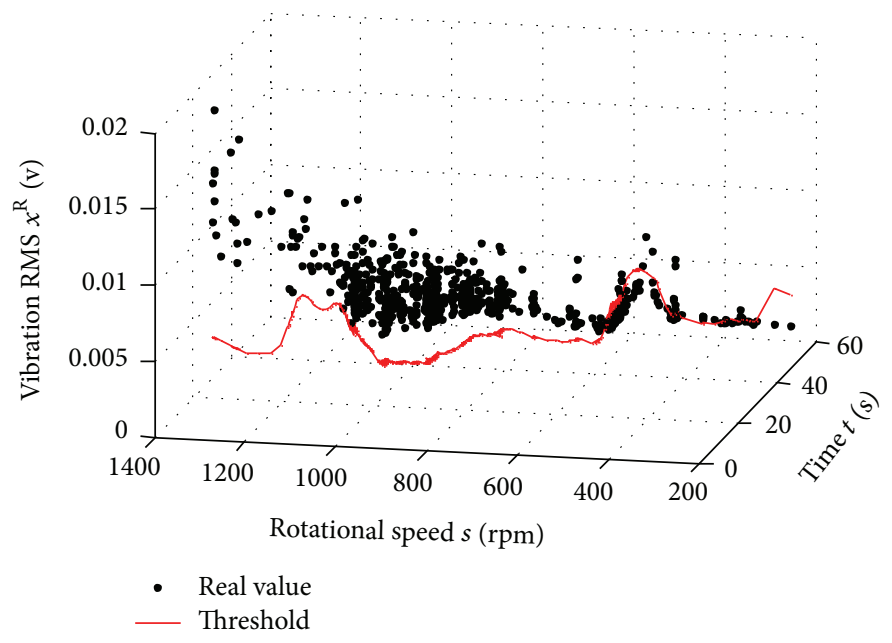

(a)

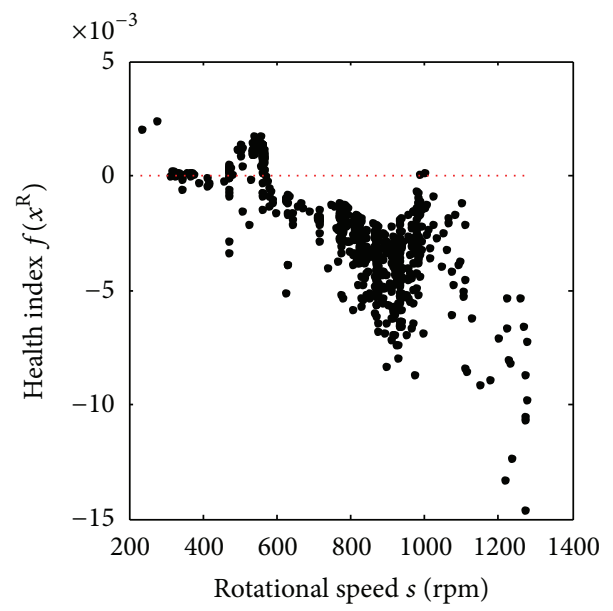

(b)

FIGURE 10: The monitoring results of a faulty bearing during Test 14.

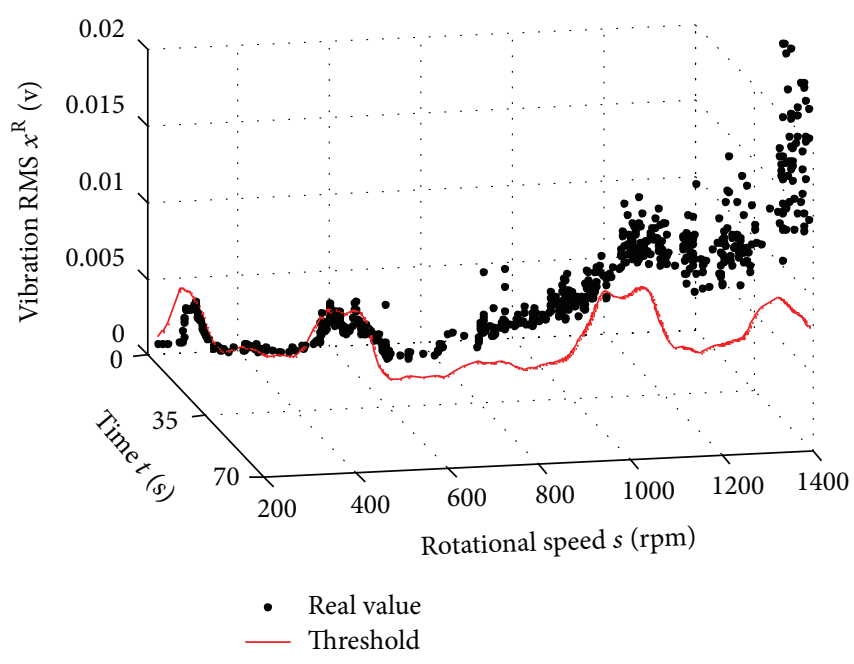

(a)

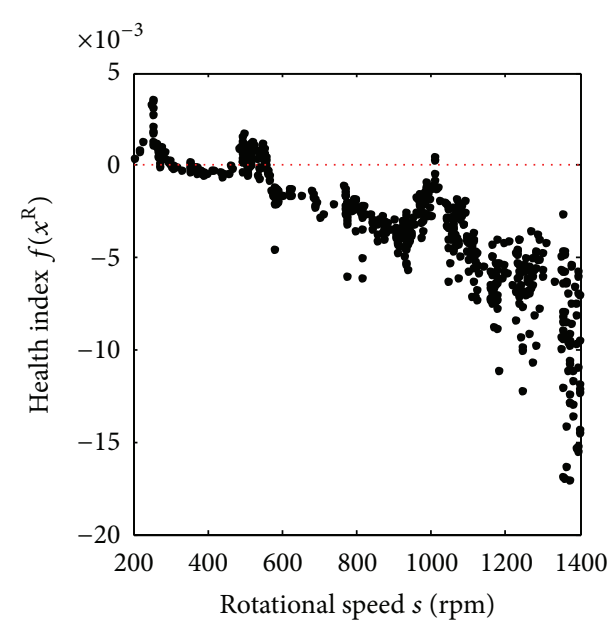

(b)

FIgURE 11: The monitoring results of a faulty bearing during Test 16.

there are lots of samples that take RMS values smaller than their corresponding thresholds. The health indexes of these samples are positive as shown in Figure 10(b). These samples are false positives. It should be noted that most of these false positives are in case where the rotational speeds are lower than $570 \mathrm{rpm}$. But there are only 2 false positives when the rotational speeds are higher than $570 \mathrm{rpm}$. These 2 false positives are at around $990 \mathrm{rpm}$. The total number of the samples whose speeds are higher than $570 \mathrm{rpm}$ is 521 . Thus the false positive rate is $2 / 521 \approx 0.004$ when rotational speeds take values in the interval [570 1400] rpm.

Figure 11 shows the monitoring results of a faulty bearing during Test 16. The rotational speeds of the tested bearing increased from $200 \mathrm{rpm}$ to $1400 \mathrm{rpm}$ in 68.2 seconds. It can be seen that there are lots of false positives where the rotational speeds are lower than $558 \mathrm{rpm}$. Most samples exceed their thresholds when the rotational speeds are higher than $558 \mathrm{rpm}$ except 2 samples at around $1009 \mathrm{rpm}$. The total number of the samples whose speeds are higher than $558 \mathrm{rpm}$ is 502 . Thus the false positive rate is $2 / 502 \approx 0.004$ when rotational speeds take values in the interval [558 1400] rpm.

The above monitoring results show that the proposed method has small false negatives and false positives are focused on the low rotational speed cases. Monitoring results of other tests show similar results. It is safe to reach the conclusion according to all the monitoring results that the proposed method is effective for the bearings of the test rig when the rotational speeds are beyond $600 \mathrm{rpm}$.

3.3. Discussions. As introduced in Section 1, the effects of bearing faults can be mainly considered as additive load fluctuations that are caused by the impact of rolling elements 
when passing through the defective zone. But at low rotational speeds the impact energy caused by defect is small and is severely attenuated though the propagation from the impact source and the accelerometers [20]. Future research efforts can be put on solving the problem at low rotating speed by extracting more effective features to replace the vibration RMS or using acoustic emission sensors.

The varying operation conditions of bearings involve not only varying rotational speeds but also varying loads. The adaptive parameters of the GTM are multivariable functions of rotational speed and load. If more than one healthy feature is extracted, these features will also be the variables of these functions. Health monitoring of bearings under varying rotational speeds and loads with multifeatures deserves research efforts.

\section{Conclusions}

We have proposed a health monitoring method that is adaptive to varying operation conditions. Adaptive GTM is selected as the basis of this method. RVMs are selected for regression of the relationships between the adaptive parameters of the monitoring model and the condition parameter, particularly the rotational speed of bearings. Since the monitoring model is a continuous function of rotational speed, it is applicable at any rotational speed. This method is applied to the health monitoring of bearings. The results show that monitoring errors are mainly focused on low rotational speed cases and the proposed method can effectively detect bearing faults as long as the rotational speed is higher than $600 \mathrm{rpm}$.

\section{Conflict of Interests}

The authors declare that there is no conflict of interests regarding the publication of this paper.

\section{Acknowledgments}

This work was supported by the National Natural Science Foundation of China [Grant no. 51105366] and the Research Project of National University of Defense Technology [Grant no. JC12-03-02].

\section{References}

[1] L. Renaudin, F. Bonnardot, O. Musy, J. B. Doray, and D. Rémond, "Natural roller bearing fault detection by angular measurement of true instantaneous angular speed," Mechanical Systems and Signal Processing, vol. 24, no. 7, pp. 1998-2011, 2010.

[2] R. B. Randall, Vibration-Based Condition Monitoring: Industrial, Aerospace and aAutomotive Applications, John Wiley and Sons, 2011.

[3] H. Li, Y. Zhang, and H. Zheng, "Gear fault detection and diagnosis under speed-up condition based on order cepstrum and radial basis function neural network," Journal of Mechanical Science and Technology, vol. 23, no. 10, pp. 2780-2789, 2009.

[4] S. Conforto and T. D. Alessio, "Spectral analysis for nonstationary signals from mechanical measurements: a parametric approach," Mechanical Systems and Signal Processing, vol. 13, no. 3, pp. 395-411, 1999.
[5] J. Lin and L. Qu, "Feature extraction based on Morlet wavelet and its application for mechanical fault diagnosis," Journal of Sound and Vibration, vol. 234, no. 1, pp. 135-148, 2000.

[6] H.-Q. Wang, W. Hou, G. Tang, H.-F. Yuan, Q.-L. Zhao, and $\mathrm{X}$. Cao, "Fault detection enhancement in rolling element bearings via peak-based multiscale decomposition and envelope demodulation," Mathematical Problems in Engineering, vol. 2014, Article ID 329458, 11 pages, 2014.

[7] J. W. Lee, J. D. Kim, C. B. Yun, J. H. Yi, and J. M. Shim, "Healthmonitoring method for bridges under ordinary traffic loadings," Journal of Sound and Vibration, vol. 257, no. 2, pp. 247-264, 2002.

[8] J.-S. Cheng, D.-J. Yu, and Y. Yang, "Research on the intrinsic mode function (IMF) criterion in EMD method," Mechanical Systems and Signal Processing, vol. 20, no. 4, pp. 817-824, 2006.

[9] W. Yang and P. J. Tavner, "Empirical mode decomposition, an adaptive approach for interpreting shaft vibratory signals of large rotating machinery," Journal of Sound and Vibration, vol. 321, no. 3-5, pp. 1144-1170, 2009.

[10] J.-D. Wu, M. R. Bai, F.-C. Su, and C.-W. Huang, "An expert system for the diagnosis of faults in rotating machinery using adaptive order-tracking algorithm," Expert Systems with Applications, vol. 36, no. 3, pp. 5424-5431, 2009.

[11] M. Lebold, K. McClintic, R. Campbell, C. Byington, K. Maynard, and N. Enveloping, "Review of vibration analysis methods for gearbox diagnostics and prognostics," in Proceedings of the 54th Meeting of the Society for Machinery Failure Prevention Technology, pp. 623-634, Virginia Beach, Va, USA, May 2000.

[12] F. di Maio, K. L. Tsui, and E. Zio, "Combining Relevance Vector Machines and exponential regression for bearing residual life estimation," Mechanical Systems and Signal Processing, vol. 31, pp. 405-427, 2012.

[13] N. G. Gebraeel, A. Elwany, and J. Pan, "Residual life predictions in the absence of prior degradation knowledge," IEEE Transactions on Reliability, vol. 58, no. 1, pp. 106-117, 2009.

[14] Z. Yang, P. K. Wong, C. M. Vong, J. Zhong, and J. J. Y. Liang, "Simultaneous-fault diagnosis of gas turbine generator systems using a pairwise-coupled probabilistic classifier," Mathematical Problems in Engineering, vol. 2013, Article ID 827128, 14 pages, 2013.

[15] C.-M. Vong, P.-K. Wong, W.-F. Ip, and C.-C. Chiu, "Simultaneous-fault diagnosis of automotive engine ignition systems using prior domain knowledge and relevance vector machine," Mathematical Problems in Engineering, vol. 2013, Article ID 974862, 19 pages, 2013.

[16] L. Liu and Z. Ding, "Modeling analysis of power transformer fault diagnosis based on improved relevance vector machine," Mathematical Problems in Engineering, vol. 2013, Article ID 636374, 6 pages, 2013.

[17] L. Hu, N. Hu, X. Zhang, F. Gu, and M. Gao, "Novelty detection methods for online health monitoring and post data analysis of turbopumps," Journal of Mechanical Science and Technology, vol. 27, no. 7, pp. 1933-1942, 2013.

[18] M. E. Tipping, "Bayesian inference: an introduction to principles and practice in machine learning," in Advanced Lectures on Machine Learning, O. Bousquet, U. von Luxburg, and G. Rätsch, Eds., pp. 41-62, Springer, Berlin, Germany, 2004.

[19] M. E. Tipping, "SparseBays: An Efficient Matlab Implementation of the Sparse Bayesian Modelling Algorithm (Version 2.0)," March, 2009, http://www.relevancevector.com.

[20] A. Widodo, B.-S. Yang, E. Y. Kim, A. C. C. Tan, and J. Mathew, "Fault diagnosis of low speed bearing based on acoustic emission signal and multi-class relevance vector machine," Nondestructive Testing and Evaluation, vol. 24, no. 4, pp. 313328, 2009. 


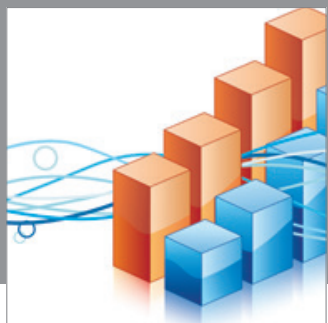

Advances in

Operations Research

mansans

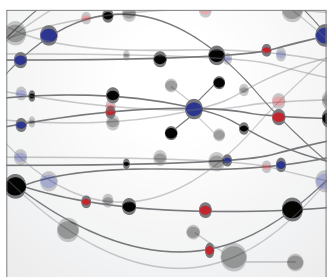

The Scientific World Journal
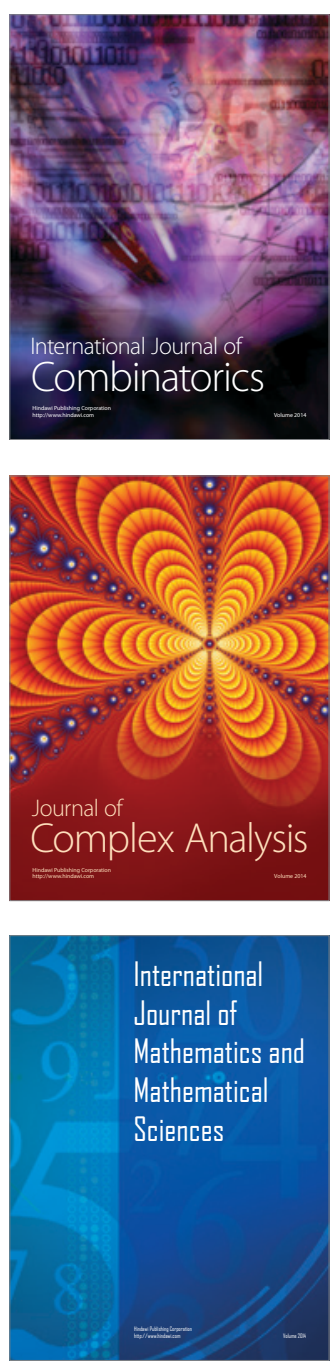
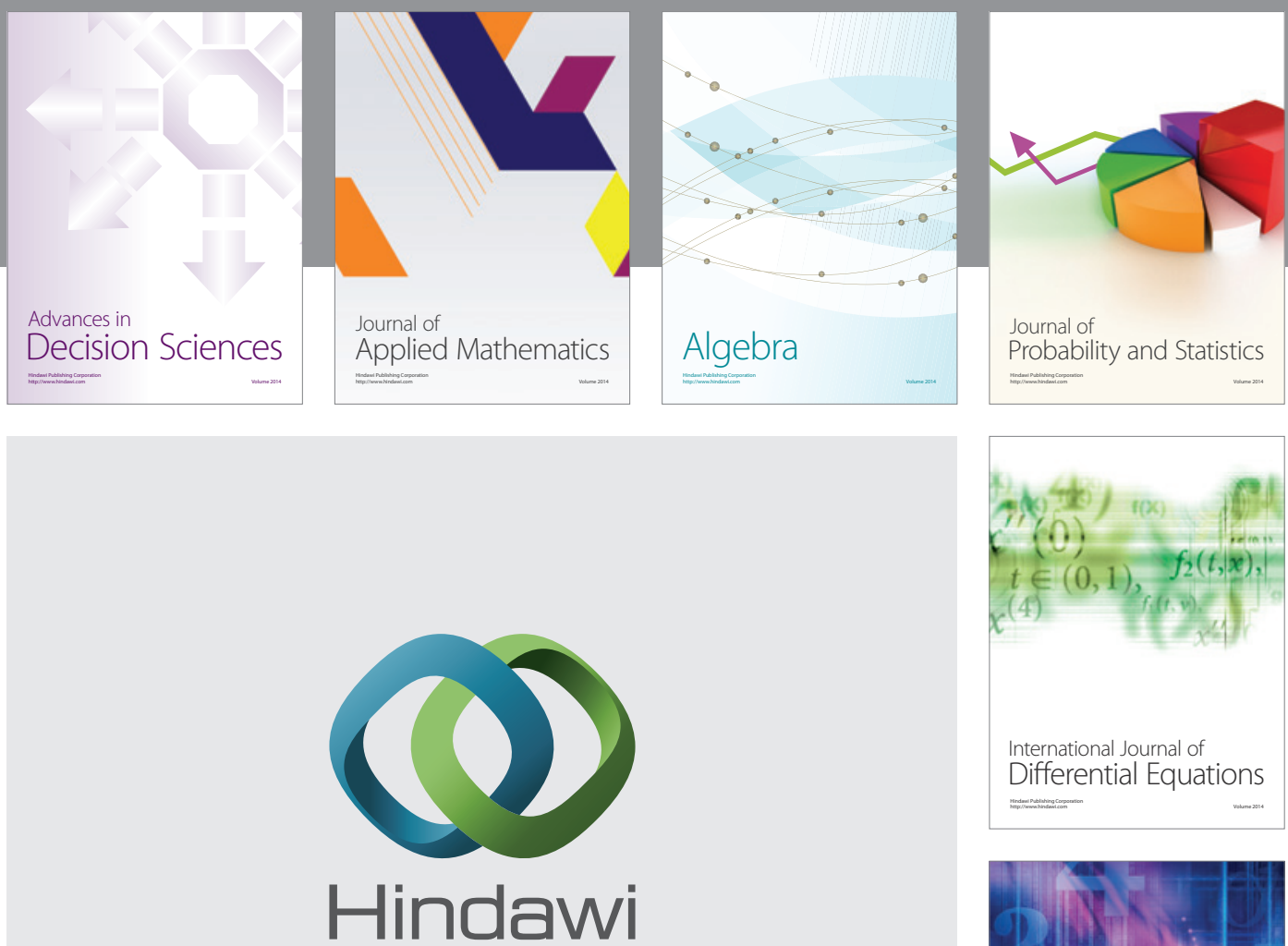

Submit your manuscripts at http://www.hindawi.com
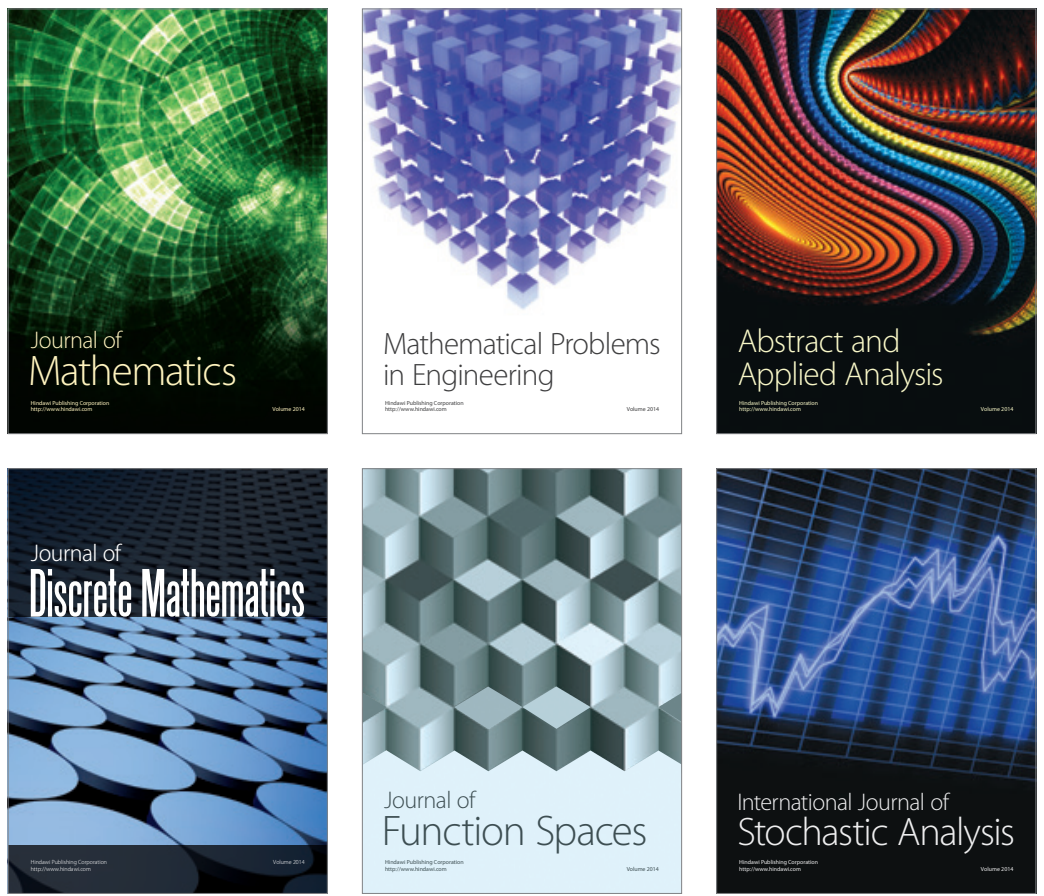

Journal of

Function Spaces

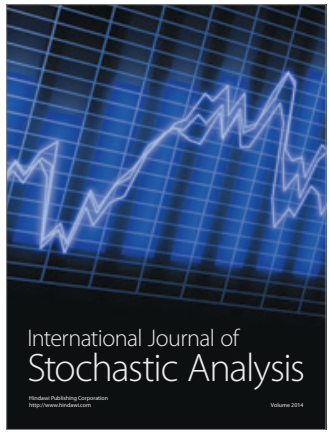

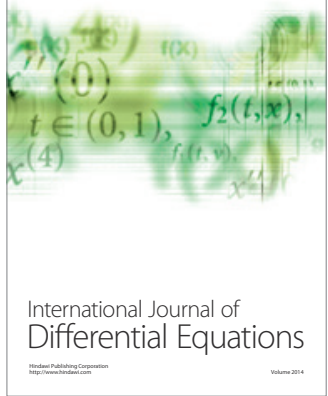
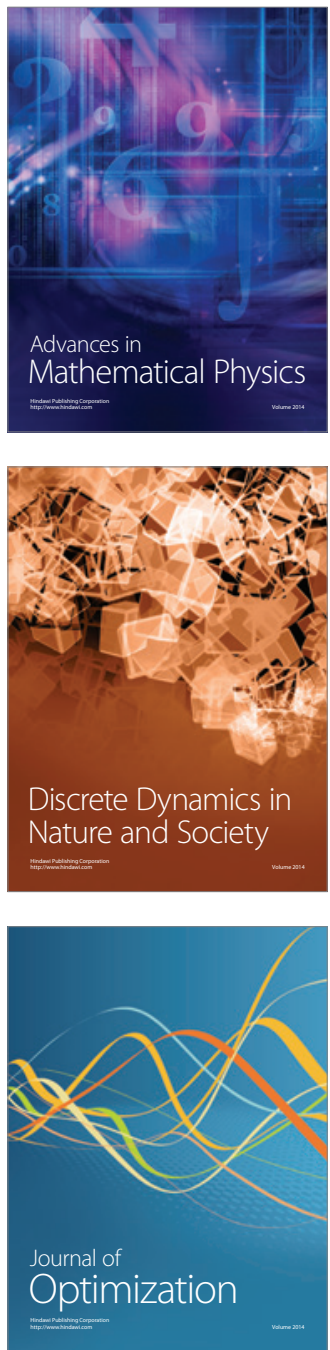